\title{
Level of Stress and Coping Strategies among Nursing Students at Damanhour University, Egypt
}

\author{
Abeer Abd El-Aziz Mohamed Madian ${ }^{1, *}$, Maha Mahmoud Abdelaziz ${ }^{2}$, Hend Abo Elsoud Ahmed ${ }^{3}$ \\ ${ }^{1}$ Community Health Nursing, Faculty of Nursing, Damanhour University, Egypt \\ ${ }^{2}$ Community Health Nursing, Faculty of Nursing. Zagazig University, Egypt \\ ${ }^{3}$ Nursing Education, Faculty of Nursing, Damanhour University, Egypt \\ *Corresponding author: abeer.madian@nur.dmu.edu.eg
}

Received April 17, 2019; Revised May 21, 2019; Accepted July 02, 2019

\begin{abstract}
Stress has been recognized as a 20th century disease and donates to health problems worldwide, nursing students are exposed to numerous stressors during their studies and clinical training. Determining stress and coping strategies among them will have significant consequences for the nursing career. Aims: to illustrate level of stress and identify coping strategies among nursing students at Damanhour University, Egypt. Research design: A descriptive cross-sectional study was used. Setting: the study was conducted at faculty of nursing, Damanhour University, Egypt. Subjects and methods: A systematic random sampling of 400 nursing students at academic year 2018-2019. Results: Students perceived moderate level of stress, most commonly attributed to transportations and academic. The most frequently used coping mechanism was religious and acceptance. The study found that gender and living with family are good predictors of coping strategies. Conclusion: The nursing students were exposed to moderate stress due to different stressors. In response, they frequently used different strategies for coping and recommended the need for stress management programs and the provision of suitable support.
\end{abstract}

Keywords: stress, coping strategies, student, nursing

Cite This Article: Abeer Abd El-Aziz Mohamed Madian, Maha Mahmoud Abdelaziz, and Hend Abo Elsoud Ahmed, "Level of Stress and Coping Strategies among Nursing Students at Damanhour University, Egypt." American Journal of Nursing Research, vol. 7, no. 5 (2019): 684-696. doi: 10.12691/ajnr-7-5-3.

\section{Introduction}

Stress is a global phenomenon of modern life styles and has been found to have bad health impact and unfavorably affect the learning of students as well as it is acknowledged as one of the most important issues in the modern world. In addition, stress refers to a dynamic interaction between the individual and the environment. In this interaction, demands, limitations and opportunities related to work may be perceived as threatening to surpass the individual's resources and skills. In case of disarrangement, this interaction may lead to cognitive, emotional and behavioral alterations [1,2].

During nursing education and training, nursing students are frequently exposed to various stressors which may directly or indirectly impede their learning, performance and overall well-being of their body and mind. Moreover, the practical components of the educational program made the program even more stressful than other programs [3]. Furthermore, Nursing is an emotionally demanding and highly stressful profession as nurses have to deal with different people, in various situations and different times of the day. During their everyday work, they have to cope with extremely emotionally charged and rapidly changing circumstances. In addition, students through nursing training face both physical and psychosocial risks because they have clinical duties related to patient care in clinical environments. In turn, stress is associated with organizational-level issues, such as increased absenteeism, decreased quality of work and decreased productivity $[4,5,6]$.

Three main groups of stressors have been identified: (i) academic stressors (testing and evaluation, fear of failure in training, problems with workload, etc.), (ii) clinical stressors (work, fear of making mistakes, negative responses to the death or suffering of patients, relationships with other members of the organization, etc.), and (iii) personal/social stressors (economic problems, imbalance between house work/school work, etc.) $[7,8]$.

Coping strategies are fundamental elements of nurses' stress reactions and become constant factor which important as the stressful event itself. So, nursing students requisite to cope with academic and clinical stress by removing the stressor through manipulating the environment, developing specific responses or search for diversion from the stressor $[9,10]$. Active coping strategies include an awareness of the stressor, followed by attempts to diminish the negative outcome. By contrast, avoidant coping is characterized by ignoring the issue, often resulting in activities that help in the denial of the problem (e.g., sleeping and isolating). Everyone employs one or more coping strategies to overcome stress, but it may not 
be effective and suitable to deal with the situation. Effective coping strategies help students to achieve markedly better in regards to their studies and aid in relieving their stress [11].

\subsection{Significance of Study}

Diverse studies accompanied worldwide stated the prevalence of stress among medical students ranging from $30 \%$ to $78 \%$ [12]. Additionally, study done in Egypt (2011) on nursing students' revealed that prevalence of high stress level; anxiety and depression were 40.2\%, 46.6\% and $27.9 \%$, respectively [13]. Learning to cope with stress is a beneficial skill for a nursing career and life ahead. By setting priorities, planning ahead and by organizing, self, one can minimize the impact of stress. Some degree of studies have investigated the perception of stress and coping strategies among Egyptian nursing students.

\subsection{Aim}

To illustrate level of stress and identify coping strategies among nursing students at Damanhour University, Egypt.

\subsection{Research Question}

What are level of stress and coping strategies experienced by the nursing students at Damanhour University, Egypt?

\section{Material and Methods}

\subsection{Research Design}

A descriptive cross sectional study design was adopted in order to fulfill the current study.

\subsection{Setting}

The study was conducted in faculty of nursing, Damanhour University, Egypt.

\subsection{Subjects}

The target populations for this research were nursing students who were studying in the above mentioned setting during the academic year (2018-2019) who are enrolled in first, second, third and fourth year.

\subsection{Sample}

The total numbers of nursing students in year 2018/2019 were 1131 students. The students at each level were chosen using a systematic random sampling technique from first to fourth academic years.

\section{Sample size}

According to Epi Info 7 sample size estimation program using the following parameter:

1. Population size (1131 students)

2. Expected frequency (50\%)
3. Margin of error (5\%)

4. Confidence co. efficient (95\%)

5. Minimum sample size (400 students)

The students will be selected from the previously selected setting according to the following table (Table 1);

Table 1. Sample size estimation table

\begin{tabular}{|c|c|c|}
\hline $\begin{array}{c}\text { Student's } \\
\text { grade/level }\end{array}$ & $\begin{array}{c}\text { Total number of } \\
\text { students /grade or level }\end{array}$ & Proposed sample size \\
\hline First year & 228 & $(228 \times 400) \div 1131=81$ \\
\hline Second year & 328 & $(328 \times 400) \div 1131=116$ \\
\hline Third year & 338 & $(338 \times 400) \div 1131=120$ \\
\hline Fourth year & 237 & $(237 \times 400) \div 1131=84$ \\
\hline Total & $\mathbf{1 1 3 1}$ & $\mathbf{4 0 0}$ \\
\hline
\end{tabular}

\subsection{Inclusion Criteria}

- Boys and girls students

- Who willing to participate in the study

- Free from chronic diseases.

\subsection{Data Collection Tools}

Data was collected using the following study tools

Tool (I): Students profile structured self-administered questionnaire:

This was developed by the researchers after reviewing the recent literature to collect necessary data included the following parts:

Part I: Personnel and demographic data: It included age, academic year, gender, marital status, place of residence, availability of playing time and recreational activities, coffee intake and smoking.

Part II: Socio demographic data of students 'parents: It included parent's educational level, parent's occupation, live with family or not, time spends in transportation to faculty and family income.

Part III: Working and academic experience: It included the students working hours, working in night shifts, working in multiple shifts, academic and job satisfaction

Part IV: Academic performance of students during last term.

Part V: Health profile of students: It included the following:

- Presence of health problems such as allergy in the nasal sinuses, headache, abdominal pain and blurred vision.

- Consequences of identified stressors like anxiety, depression, sleeping disorders, difficult in paying attention in class and low self-esteem.

Part VI: Anthropometric measurements:

Anthropometric measurements were estimated using standardized procedures and calibrated equipment. Height was measured using a non- stretching measuring tape in centimeters to the nearest $0.1 \mathrm{~cm}$. Weight was measured using a standardized weighing scale in kilograms to the nearest $0.5 \mathrm{~kg}$ and Body Mass Index $\left(\mathrm{Kg} / \mathrm{m}^{2}\right)$ was estimated using standardized weighting and height equation. The calculated Body Mass Index (BMI) was compared with the reference value and interpreted as following was estimated using standardized weighting and height equation of WHO 2011 as following:- 
Less than $18.5 \mathrm{~kg} / \mathrm{cm}^{2}$ (underweight)

$18.5-24.9 \mathrm{~kg} / \mathrm{cm}^{2} \quad$ (normal weight)

$25.0-29.9 \mathrm{~kg} / \mathrm{cm}^{2} \quad$ (overweight)

$30.0-34.9 \mathrm{~kg} / \mathrm{cm}^{2} \quad$ (obesity) [14].

Tool (II): Stress assessment scale questionnaire

It was developed by the researchers after reviewing of literatures to assess the types and level of stress among nursing academic students. A total of 64 potential stressors were listed and grouped as academic, interpersonal, psychosocial and transportation. The students were asked to indicate if any of the stressors had been affecting them. For each potential stressor, the scored frequency of occurrence was classified as never, rarely/sometimes, and often/always and is scored as 0,1 , and 2, respectively. The total score of stress level was obtained for each student (0-128). Percent of the total stress score was calculated as follows:-

$$
\begin{array}{ll}
\text { Mild }<64 & (<50 \%) \\
\text { Moderate 64 <96 } & (50-<75 \%) \\
\text { Sever } \geq 96 & (\geq 75 \%)
\end{array}
$$

Tool III: Coping Orientation to Problems Experienced (COPE) inventory questionnaire

This tool was used to find out the coping responses of the nursing students. The tool was developed by Dr. Charles Carver, to determine the response of people when confronted with difficult or stressful events in their lives [15]. It composed of 24 items and assessed the following scales: Self-distraction - items 1 and 19; active coping - items 2 and 7; denial - items 3 and 8; ; use of emotional support - items 5 and 15; behavioral disengagement - items 6 and 16; venting - item 9 ; use of instrumental support - items 10 and 23; positive reframing - items 12 and 17; self-blame - items 13 and 26, planning - items 14 and 25; humor - item 18; acceptance - items 20 and 24; religion - items 22 and 27. the scored frequency of occurrence was classified as; usually don't do this at all, , usually do this a little bit, usually do this a medium amount and usually do this a lot and is scored as $0,1,2$ and 3 respectively. The total coping strategies score were obtained for each student (0-72). Percent of the total score was calculated as follows:-

$\begin{array}{ll}\text { Poor }<36 & (<50 \%) \\ \text { Fair 36 }<54 & (50-<75 \%) \\ \text { Good } \geq 54 & (\geq 75 \%)\end{array}$

\section{Methods of Data Collection}

\subsection{Ethical Considerations}

- The students were asked for an oral consent to participate in the study.

- Oral consent obtained from the head of scientific departments that included in the study after explanation of the aim of the study and assure them that collected data will be used only for the study purpose.

- Confidentiality of students' response was guaranteed.

\subsection{Administrative Process}

- Official letters to the dean of Faculty of Nursing, University of Damanhour to inform about the study aim and to seek permission to conduct the study in faculty.
- Meetings were held with the head of the scientific departments of first, second, third and fourth year to explain the aim of the study, set the date and time of data collection, assure them that collected data were used only for the study purpose, and to gain their approval and cooperation during data collection.

\subsection{Development of Study Tools}

Tool (I) and (II) were developed by the researchers after reviewing recent literatures in order to collect the obligatory data from the undergraduates' students.

\subsection{Validity and Reliability}

- Tool (I) and (II) were revised by Jury composed of (three) experts in the field of community health nursing and nursing education for content validity and their recommended modifications were done accordingly.

- Tool (III) was translated in to Arabic language, revised by Jury composed of (three) experts in order to ascertain that language is clear.

- Reliability of the Tool (I) and (II) were assessed using Cronbach's Alpha Coefficient: tool I (.895); and tool II (.924).

\subsection{Pilot Study}

The Pilot study was carried out on a sample of 40 students who were excluded from the study. Accordingly, minor changes were made. The data obtained from the pilot study were analyzed. Based on the findings of the pilot study, some questions were clarified and few others added.

\subsection{Field Work}

- Students were invited to complete a self-administered questionnaire; the questionnaires were administered and collected by the researchers. Students were given detailed instructions on how to complete the questionnaire and informed about the aim of the study.

- At the beginning of the meeting with students, the researchers were introduced themselves, clarify the purpose of study, and ensure the anonymity and confidentiality of the collected data.

- Weight was measured by a standardized weighing scale, in kilograms to the nearest $0.5 \mathrm{~kg}$ and height was measured by a non- stretching measuring tape, in centimeters to the nearest $0.1 \mathrm{~cm}$, to calculate (BMI) based on WHO 2011 [14]. The structured questionnaire time took approximately from 10 to 15 minutes for each student.

- Data about students' academic performance were obtained from their faculty records in the last term.

- Data was collected by the researchers over a period of 2 months at the academic year 2018/2019.

\section{Statistical Analysis}

- After data collection, it was coded and transferred into especially designed format to be suitable for computer feeding. 
- Data was entered into and analyzed using the statistical package of social science (SPSS) version 20.

- After data entry, data was checked and revised through frequency analysis, cross tabulation, and manual revision to discover any error during data entry.

- Variables were analyzed using the descriptive statistics which included: percentages, frequencies, range (minimum and maximum), arithmetic mean, and standard deviation (SD).

- The level of significance selected for this study was p $\leq 0.05$.

- Chi square test $\left(\mathrm{X}^{2}\right)$ was used for testing the relationship between categorical variables.

- ANOVA test(F) was used to compare between sample means for quantitative data with normal distribution

- Linear Regression model was used to indicate the predictors of stress and coping strategies among students, the model was statistically significant $(\mathrm{p} \leq 0.05)$.

- Graphs were done for data visualization by using Microsoft Excel program.

\section{Results}

Table 2 displays that the student's age ranged from 19 to 23 years old with a mean of $20.7 \pm 1.1$ years. It was observed that the majority of students in the current study were single females (91.4\% and $84.5 \%$ respectively).More than two thirds $(67.8 \%)$ of them were urban residence, nearly less than half (41.5\%) did not practice any recreational activities, furthermore about half (48\%) of them had less than $2 \mathrm{hrs} /$ day to practice any recreational activities and only16\% of them had more than 4 hrs / day for play and recreational activities, whereas almost all students didn't smoke and less than half (40.7\%) had daily coffee intake.

Table 3 illustrates that nearly half $(46.2 \%, 48.8 \%)$ of the father and mother respectively had obtain diploma education. Regarding fathers' job, it was observed that more than half $(59.8 \%)$ of them were employees while about one fifth (18\%) had free work compared to approximately less than one quarter (23\%) of mothers were working and more than three quarters (77\%) of them were house wife. Majority of students living with their family and more than half $(51.5 \%)$ of them spend 50 minutes or more in transportation to faculty. In relation to family income it was observed that nearly half (45.8\%) of the studied students had enough income without saving.

Table 4 reveals that, nearly one fifth $(18.5 \%)$ of the students working all the year, among those who working around two thirds $(67.7 \%)$ of them reported that they work more than 6 hours /day while more than half (52.6\%) of them had more than one shift / day and nearly less than three quarters (71.7\%)of them had night shifts.

With regard to the student's academic performance during the last term; Figure 1 portrays that, around one third $(32.3 \%, 34.3 \%)$ of students obtain excellent and very good score respectively, while only $5.4 \%$ and $1.5 \%$ obtain pass and poor score respectively .

Table 5 portrays that minority $(7.0 \%)$ of the students complains of health problem; among them, around half (42.9\%) where complains of allergy in the nasal sinuses, followed by headache that was reported by around one third (32.1\%) of them. Besides, more than half (57\%) of the students suffering from problems of identified stressors, the majority of them complains of anxiety, depression, sleeping disorders, and difficult in paying attention in the class (98.7\%, 83.8\%, 81.6\% and 67.5\% respectively).

Figure 2 display Body Mass Index (BMI) classification among students, about tenth $(10.4 \%)$, of the students were underweight while slightly more than one third (35.3\%) were normal weight and (48.5\% and 5.8\%) were overweight and obese respectively.

Table 2. Distribution of undergraduates' students along with their personal and demographic characteristics

\begin{tabular}{|c|c|c|}
\hline Personal and demographic characteristics & $\begin{array}{c}\text { Frequency } \\
(400)\end{array}$ & $\%$ \\
\hline \multicolumn{3}{|l|}{ Age (years) } \\
\hline-19 & 62 & 15.5 \\
\hline-20 & 97 & 24.3 \\
\hline-21 & 156 & 39.0 \\
\hline - 22 and more & 85 & 21.2 \\
\hline Mean \pm SD & \multicolumn{2}{|c|}{$20.7 \pm 1.1$} \\
\hline Min -Max & \multicolumn{2}{|c|}{$19-23$} \\
\hline \multicolumn{3}{|l|}{ Academic year } \\
\hline - $1^{\text {st }}$ year & 81 & 20.3 \\
\hline$-2^{\text {nd }}$ year & 116 & 29.0 \\
\hline$-3^{\text {rd }}$ year & 120 & 30.0 \\
\hline$-4^{\text {th }}$ year & 84 & 21.0 \\
\hline \multicolumn{3}{|l|}{ Gender } \\
\hline Male & 62 & 15.5 \\
\hline Female & 338 & 84.5 \\
\hline \multicolumn{3}{|l|}{ Marital status } \\
\hline - Single & 366 & 91.4 \\
\hline - Married & 33 & 8.3 \\
\hline - Divorced & 1 & .3 \\
\hline \multicolumn{3}{|l|}{ Place of residence } \\
\hline Rural & 129 & 32.2 \\
\hline Urban & 271 & 67.8 \\
\hline \multicolumn{3}{|l|}{ Availability of recreational activities } \\
\hline No & 166 & 41.5 \\
\hline Yes & 234 & 58.5 \\
\hline \multicolumn{3}{|l|}{ Hours of recreational activities / day } \\
\hline Less than $2 \mathrm{~h}$ / day & 192 & 48.0 \\
\hline 2-4 h /day & 144 & 36.0 \\
\hline More than 4 h / day & 64 & 16.0 \\
\hline \multicolumn{3}{|l|}{ Daily coffee intake } \\
\hline No & 237 & 59.3 \\
\hline Yes & 163 & 40.7 \\
\hline \multicolumn{3}{|l|}{ Smoking } \\
\hline No & 396 & 99.0 \\
\hline Yes & 4 & 1.0 \\
\hline
\end{tabular}


Table 3. Distribution of students' parents along with their socio demographic characteristics

\begin{tabular}{|l|c|c|}
\hline Parents socio demographic characteristics & $\begin{array}{c}\text { Frequency } \\
\text { (400) }\end{array}$ & $\mathbf{\%}$ \\
\hline Father education & & \\
\hline Illiterate & 33 & 8.2 \\
\hline Primary & 25 & 6.3 \\
\hline Secondary & 31 & 7.8 \\
\hline Diploma & 185 & 46.2 \\
\hline University & 126 & 31.5 \\
\hline Father job & & \\
\hline Retired & 40 & 10.0 \\
\hline Manual work & 37 & 9.2 \\
\hline Employee & 239 & 59.8 \\
\hline Free work & 72 & 18.0 \\
\hline Private work & 12 & 3.0 \\
\hline Mother education & & \\
\hline Illiterate & 72 & 18.0 \\
\hline Primary & 30 & 7.5 \\
\hline Secondary & 30 & 7.5 \\
\hline Diploma & 195 & 48.8 \\
\hline University & 73 & 18.2 \\
\hline Mother work & & \\
\hline House wife & 308 & 77.0 \\
\hline Working & 92 & 23.0 \\
\hline Living with family & 376 & 94.0 \\
\hline Yes & 24 & 6.0 \\
\hline No & 1189 & \\
\hline Time spends in transportation to faculty & 51.5 \\
\hline 50 minutes or more & 76 & 29.5 \\
\hline 30 -50 minutes & & 19 \\
\hline$<30$ or less & & \\
\hline Family income & & \\
\hline Enough and save & & \\
\hline Enough and no save & & \\
\hline Not enough & & \\
\hline
\end{tabular}

Table 4. Distribution of undergraduates' students along with their working and academic experience

\begin{tabular}{|l|c|c|}
\hline Working and academic experience & Frequency ( 400) & \% \\
\hline Student Working experience & & \\
\hline Working during vocation only & 78 & 19.5 \\
\hline Working all year & 74 & 18.5 \\
\hline Didn't work & 248 & 62.0 \\
\hline Working hours/day & $\mathbf{n ( 1 5 2 )}$ & \\
\hline 6 hours & 49 & 32.3 \\
\hline More than 6 hours & 103 & 67.7 \\
\hline Have night shift & & \\
\hline No & 43 & 28.3 \\
\hline Yes & 109 & 71.7 \\
\hline Have more than one shift / day & & \\
\hline No & 72 & 47.4 \\
\hline Yes & 80 & 52.6 \\
\hline Academic and job satisfaction & & \\
\hline Satisfied & 260 & 65.0 \\
\hline Not satisfied & 140 & 35.0 \\
\hline
\end{tabular}

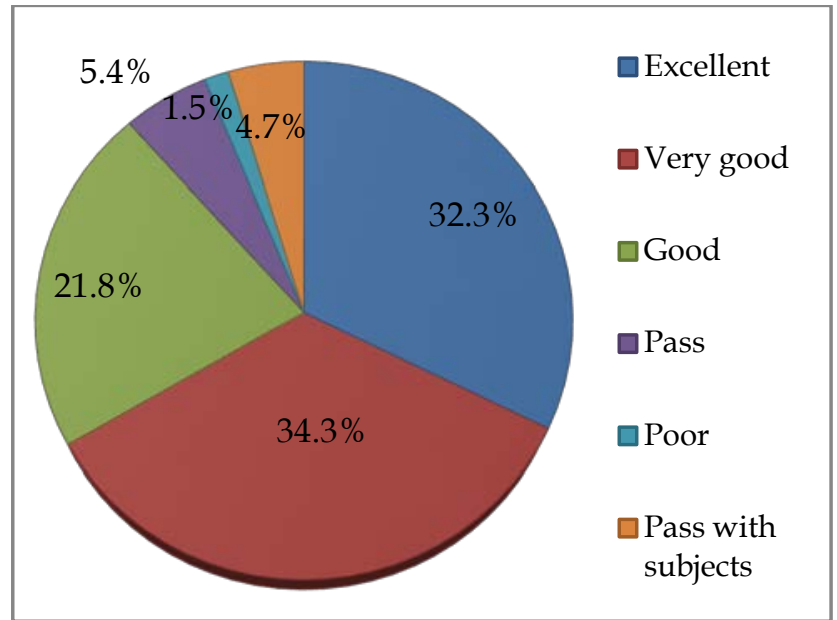

Figure 1. Academic performance during last term among undergraduates' students

Table 5. Distribution of undergraduates' students along with their health profile

\begin{tabular}{|l|c|c|}
\hline Health profile & frequency & \% \\
\hline Presence of health problem & & \\
\hline No & 372 & 93.0 \\
\hline Yes & 28 & 7.0 \\
\hline \#Type of health problem & $\mathrm{n}(28)$ & \\
\hline Allergy in the nasal sinuses & 12 & 42.9 \\
\hline Headache & 9 & 32.1 \\
\hline Abdominal pain & 10 & 35.7 \\
\hline Blurred vision & 8 & 28.6 \\
\hline Consequences of identified stressors & & \\
\hline No & 172 & 43.0 \\
\hline Yes & 228 & 57.0 \\
\hline \# types & & \\
\hline Anxiety & 225 & 98.7 \\
\hline Depression & 191 & 83.8 \\
\hline Sleeping disorders & 186 & 81.6 \\
\hline Difficult in paying attention in class & 154 & 67.5 \\
\hline Low self esteem & 137 & 60.1 \\
\hline
\end{tabular}

\# More than one answer

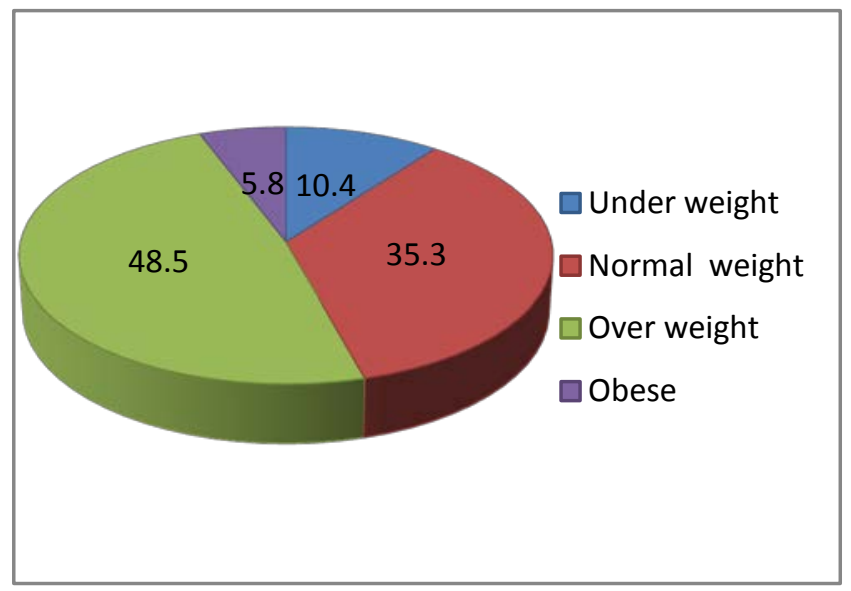

Figure 2. Body mass indexes among undergraduates's students 
Table 6. Types and mean percent of stressors perceived by undergraduates' students

\begin{tabular}{|l|c|c|c|}
\hline Stressors & Maximum allowed scores & Mean \pm SD & Mean\% \pm SD \\
\hline Academic stress & 62 & $32.9 \pm 9.6$ & $53.2 \pm 15.5$ \\
\hline Inter personal stress & 32 & $14.1 \pm 6.0$ & $44.1 \pm 18.9$ \\
\hline Social stress & 28 & $11.7 \pm 4.7$ & $42.0 \pm 16.9$ \\
\hline Stress related to Transportation & 6 & $4.2 \pm 1.7$ & $70.1 \pm 29.0$ \\
\hline Total stress & 128 & $63.1 \pm 17.9$ & $49.3 \pm 14.0$ \\
\hline
\end{tabular}

Table 7. Distribution of stress level perceived by undergraduate students

\begin{tabular}{|l|c|c|}
\hline Stressors & Frequency & $\mathbf{\%}$ \\
\hline Academic stress & & 27.8 \\
\hline Mild & 111 & 50.0 \\
\hline Moderate & 200 & 22.2 \\
\hline Severe & 89 & 27.8 \\
\hline Personal stress & & 49.8 \\
\hline Mild & 111 & 22.4 \\
\hline Moderate & 199 & 35.5 \\
\hline Severe & 90 & 42.5 \\
\hline Social stress & & 22.0 \\
\hline Mild & 142 & 31.3 \\
\hline Moderate & 170 & 37.3 \\
\hline Severe & 88 & 31.4 \\
\hline Stress related to Transportation & & \\
\hline Mild & 125 & 28.5 \\
\hline Moderate & 149 & 48.3 \\
\hline Severe & 126 & 23.2 \\
\hline Total stress & & \\
\hline Mild & 114 & \\
\hline Moderate & 193 & \\
\hline Sever & 93 & \\
\hline
\end{tabular}

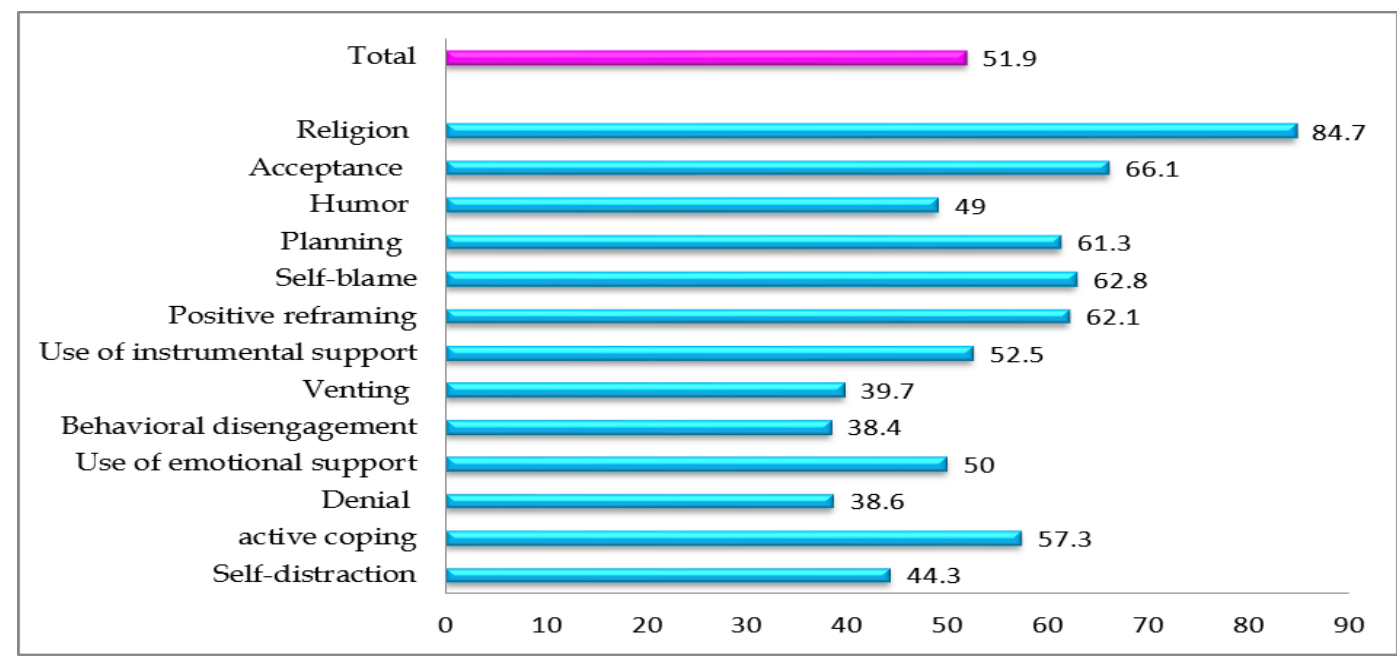

Figure 3. Coping strategies mean percent score among undergraduates' students

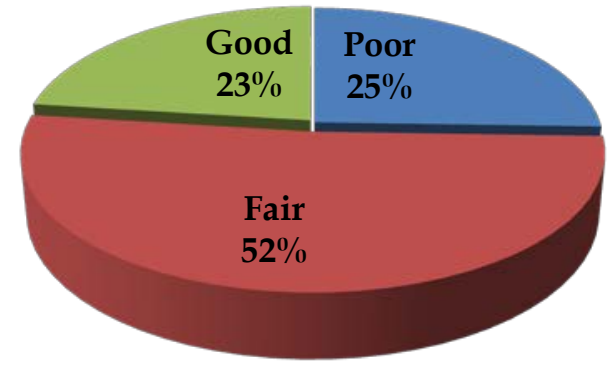

Figure 4. Coping strategies total score classification among undergraduates' students 
Table 8. Classification of stressors scores of undergraduates' students in relation to their coping strategies

\begin{tabular}{|c|c|c|c|c|c|c|c|}
\hline \multirow{2}{*}{$\begin{array}{l}\text { Stressors } \\
\text { Academic stressors }\end{array}$} & \multicolumn{6}{|c|}{ Coping Strategies } & \multirow{2}{*}{ Test of significance } \\
\hline & \multicolumn{2}{|c|}{ Poor } & \multicolumn{2}{|c|}{ Fair } & \multicolumn{2}{|c|}{ Good } & \\
\hline Mild & 44 & $39.6 \%$ & 53 & $47.7 \%$ & 14 & $12.6 \%$ & \multirow{3}{*}{$\begin{array}{l}X^{2}: 20.952 \\
P:<0.001^{*}\end{array}$} \\
\hline Moderate & 44 & $22.0 \%$ & 103 & $51.5 \%$ & 53 & $26.5 \%$ & \\
\hline Severe & 14 & $15.7 \%$ & 50 & $56.2 \%$ & 25 & $28.1 \%$ & \\
\hline \multicolumn{8}{|l|}{ Personal stressors } \\
\hline Mild & 26 & $23.4 \%$ & 62 & $55.9 \%$ & 23 & $20.7 \%$ & \multirow{3}{*}{$\begin{array}{l}X^{2}: 9.999 \\
P: 0.052^{*}\end{array}$} \\
\hline Moderate & 60 & $30.2 \%$ & 100 & $50.3 \%$ & 39 & $19.6 \%$ & \\
\hline Severe & 16 & $17.8 \%$ & 44 & $48.9 \%$ & 30 & $33.3 \%$ & \\
\hline \multicolumn{8}{|l|}{ Social stressors } \\
\hline Mild & 47 & $33.1 \%$ & 70 & $49.3 \%$ & 25 & $17.6 \%$ & \multirow{3}{*}{$\begin{array}{c}\mathrm{X}^{2}: 12.222 \\
\mathrm{P}: 0.002^{*}\end{array}$} \\
\hline Moderate & 43 & $25.3 \%$ & 84 & $49.4 \%$ & 43 & $25.3 \%$ & \\
\hline Severe & 12 & $13.6 \%$ & 52 & $59.1 \%$ & 24 & $27.3 \%$ & \\
\hline \multicolumn{8}{|c|}{ Transportation stressors } \\
\hline Mild & 45 & $36.0 \%$ & 62 & $49.6 \%$ & 18 & $14.4 \%$ & \multirow{3}{*}{$\begin{array}{l}X^{2}: 15.204 \\
P:<0.001^{*}\end{array}$} \\
\hline Moderate & 35 & $23.5 \%$ & 76 & $51.0 \%$ & 38 & $25.5 \%$ & \\
\hline Severe & 22 & $17.5 \%$ & 68 & $54.0 \%$ & 36 & $28.6 \%$ & \\
\hline \multicolumn{8}{|l|}{ Total stressors } \\
\hline Mild & 33 & $28.9 \%$ & 63 & $55.3 \%$ & 18 & $15.8 \%$ & \multirow{3}{*}{$\begin{array}{l}X^{2}: 9.133 \\
P: 0.005^{*}\end{array}$} \\
\hline Moderate & 53 & $27.5 \%$ & 95 & $49.2 \%$ & 45 & $23.3 \%$ & \\
\hline Severe & 16 & $17.2 \%$ & 48 & $51.6 \%$ & 29 & $31.2 \%$ & \\
\hline
\end{tabular}

$\mathrm{X}^{2}$ : chi-square, * significant at $\mathrm{p}$ value $\leq 0.05$.

Table 9. Distribution of students along with their socio demographic characteristics, mean stress percent and mean coping strategies percent

\begin{tabular}{|c|c|c|c|c|}
\hline Socio-demographic & Mean stress \% & Test of significance & Mean coping strategies \% & Test of significance \\
\hline \multicolumn{5}{|l|}{ Age (years) } \\
\hline-19 & $48.7 \pm 15.8$ & \multirow{4}{*}{$\begin{array}{l}\text { F:2.952 } \\
\text { P0.033* }\end{array}$} & $49.6 \pm 14.8$ & \multirow{4}{*}{$\begin{array}{l}F: 1.073 \\
P: 0.361\end{array}$} \\
\hline-20 & $47.0 \pm 13.5$ & & $53.9 \pm 17.0$ & \\
\hline-21 & $51.2 \pm 13.8$ & & $51.7 \pm 14.1$ & \\
\hline - 22 and more & $51.8 \pm 13.0$ & & $51.5 \pm 14.9$ & \\
\hline \multicolumn{5}{|l|}{ Gender } \\
\hline Male & $54.16 \pm 13.9$ & \multirow{2}{*}{$\begin{array}{c}\text { F:8.795 } \\
\text { P: } 0.003^{*}\end{array}$} & $59.4 \pm 15.3$ & \multirow{2}{*}{$\begin{array}{l}\mathrm{F}: 18.751 \\
\mathrm{P}:<0.001^{*}\end{array}$} \\
\hline Female & $48.4 \pm 13.8$ & & $50.5 \pm 14.7$ & \\
\hline \multicolumn{5}{|l|}{ Marital status } \\
\hline - Single & $49.6 \pm 13.7$ & \multirow{3}{*}{$\begin{array}{l}F: 0.917 \\
P: 0.433\end{array}$} & $52.1 \pm 15.4$ & \multirow{3}{*}{$\begin{array}{l}\text { F:0.669 } \\
\text { P:0.572 }\end{array}$} \\
\hline - Married & $45.7 \pm 10.6$ & & $51.0 \pm 13.6$ & \\
\hline - Divorced & $52.3 \pm 0.00$ & & $65.3 \pm 0.00$ & \\
\hline \multicolumn{5}{|l|}{ Place of residence } \\
\hline Rural & $48.1 \pm 16.3$ & \multirow{2}{*}{$\begin{array}{l}\mathrm{F}: 1.440 \\
\mathrm{P}: 0.231\end{array}$} & $52.0 \pm 13.0$ & \multirow{2}{*}{$\begin{array}{l}\text { F:0.006 } \\
\text { P:0.939 }\end{array}$} \\
\hline Urban & $49.9 \pm 12.7$ & & $51.8 \pm 16.0$ & \\
\hline \multicolumn{5}{|l|}{ Living with family } \\
\hline Yes & $49.5 \pm 14.1$ & \multirow{2}{*}{$\begin{array}{l}F: 1.529 \\
P: 0.217\end{array}$} & $52.2 \pm 15.3$ & \multirow{2}{*}{$\begin{array}{l}\text { F: } 2.414 \\
\text { P:0.121 }\end{array}$} \\
\hline No & $45.9 \pm 10.2$ & & $47.2 \pm 11.2$ & \\
\hline \multicolumn{5}{|l|}{ Family income } \\
\hline Enough and save & $44.3 \pm 14.9$ & \multirow{3}{*}{$\begin{array}{c}F: 21.317 \\
P:<0.001^{*}\end{array}$} & $51.3 \pm 16.0$ & \multirow{3}{*}{$\begin{array}{l}\text { F:0.239 } \\
\text { P:0.788 }\end{array}$} \\
\hline Enough and no save & $51.5 \pm 11.5$ & & $52.4 \pm 13.6$ & \\
\hline Not enough & $56.1 \pm 13.8$ & & $51.7 \pm 17.2$ & \\
\hline
\end{tabular}

F: ANOVA test, * significant at $\mathrm{p}$ value $\leq 0.05$. 
Table 10. Significant between working and academic experience among undergraduates' students with mean stress percent and mean coping strategies percent

\begin{tabular}{|c|c|c|c|c|}
\hline Working and academic experience & Mean stress \% & Test of significance & Mean strategies \% & Test of significance \\
\hline \multicolumn{5}{|l|}{ Student Working experience } \\
\hline Working during vocation & $50.1 \pm 11.6$ & \multirow{3}{*}{$\begin{array}{l}F: 0.680 \\
P: 0.507\end{array}$} & $53.1 \pm 13.6$ & \multirow{3}{*}{$\begin{array}{l}F: 0.363 \\
P: 0.696\end{array}$} \\
\hline Working all years & $50.6 \pm 15.8$ & & $51.0 \pm 18.0$ & \\
\hline Didn't work & $48.7 \pm 14.1$ & & $51.7 \pm 14.6$ & \\
\hline \multicolumn{5}{|l|}{ Working hours/day } \\
\hline 6 hours & $49.7 \pm 12.2$ & \multirow{2}{*}{$\begin{array}{l}F: 0.735 \\
P 0.0480\end{array}$} & $53.3 \pm 15.8$ & \multirow{2}{*}{$\begin{array}{l}F: 0.735 \\
P: 0.480\end{array}$} \\
\hline More than 6 hours & $50.6 \pm 14.5$ & & $51.5 \pm 16.0$ & \\
\hline \multicolumn{5}{|l|}{ Have night shift } \\
\hline No & $53.8 \pm 10.0$ & \multirow{2}{*}{$\begin{array}{c}\mathrm{F}: 3.958 \\
\mathrm{P}: 0.048^{*}\end{array}$} & $49.1 \pm 15.1$ & \multirow{2}{*}{$\begin{array}{l}F: 2.134 \\
P: 0.146\end{array}$} \\
\hline Yes & $49.0 \pm 14.8$ & & $53.3 \pm 16.1$ & \\
\hline \multicolumn{5}{|l|}{ Have more than shift } \\
\hline No & $52.4 \pm 14.4$ & \multirow{2}{*}{$\begin{array}{l}F: 2.975 \\
P: 0.087\end{array}$} & $49.7 \pm 15.6$ & \multirow{2}{*}{$\begin{array}{l}F: 3.190 \\
P: 0.076\end{array}$} \\
\hline Yes & $48.5 \pm 13.0$ & & $54.2 \pm 16.0$ & \\
\hline \multicolumn{5}{|l|}{ Academic level during the last term } \\
\hline Excellent & $47.8 \pm 15.3$ & \multirow{6}{*}{$\begin{array}{l}F: 2.019 \\
P: 0.075\end{array}$} & $50.3 \pm 15.2$ & \multirow{6}{*}{$\begin{array}{c}\mathrm{F}: 4.491 \\
\mathrm{P}: 0.001^{*}\end{array}$} \\
\hline Very good & $48.5 \pm 13.3$ & & $49.1 \pm 15.5$ & \\
\hline Good & $52.6 \pm 13.1$ & & $58.1 \pm 13.1$ & \\
\hline Pass & $46.3 \pm 10.8$ & & $51.2 \pm 11.7$ & \\
\hline Poor & $48.7 \pm 15.6$ & & $50.6 \pm 14.5$ & \\
\hline Pass with subjects & $54.0 \pm 13.0$ & & $54.7 \pm 17.1$ & \\
\hline
\end{tabular}

$\mathrm{F}:$ ANOVA test, $*$ significant at $\mathrm{p}$ value $\leq 0.05$.

Table 11. Multivariate Analysis Logistic Regression for stress level and coping strategies among undergraduates' students

\begin{tabular}{|c|c|c|c|c|c|c|c|c|}
\hline \multirow{3}{*}{ Variables } & \multicolumn{4}{|c|}{ Stress level } & \multicolumn{4}{|c|}{ Coping strategies } \\
\hline & \multirow{2}{*}{ OR } & \multirow{2}{*}{ Sig. } & \multicolumn{2}{|c|}{ 95\% Confidence Interval } & \multirow{2}{*}{ OR } & \multirow{2}{*}{ Sig. } & \multicolumn{2}{|c|}{ 95\% Confidence Interval } \\
\hline & & & Lower Bound & Upper Bound & & & Lower Bound & Upper Bound \\
\hline Academic year & .206 & .650 & -.375 & .601 & .158 & .691 & -.593 & .393 \\
\hline Age & .692 & .406 & -.903 & .365 & .206 & .650 & -.774 & .483 \\
\hline Gender & 11.301 & $.001^{*}$ & .391 & 1.485 & 12.809 & $.000 *$ & .449 & 1.535 \\
\hline Family income & 26.075 & $.000 *$ & -2.199 & -.979 & .103 & .749 & -.683 & .491 \\
\hline Living with family & 1.724 & .189 & -.258 & 1.305 & 3.794 & $.051 *$ & -.005 & 1.577 \\
\hline Health problems & 1.269 & .260 & -1.188 & .321 & .566 & .452 & -1.032 & .459 \\
\hline BMI & 3.755 & $.053 *$ & -.006 & .972 & 4.829 & $.028 *$ & -1.048 & -.060 \\
\hline Coffee intake & 3.489 & .062 & -.018 & .766 & 1.558 & .212 & -.635 & .141 \\
\hline
\end{tabular}

OR: Odds ratio, * significant at $\mathrm{p}$ value $\leq 0.05$.

Table 6 presents the ranking of stressors from the students perspective, where stress related to transportation was ranked firstly followed by academic stress, inter-personal stress and finally social stress with a mean percentage of $70.1 \pm 29.0,53.2 \pm 15.5,44.1 \pm 18.9$, and $42.0 \pm 16.9$ respectively.

Table 7 shows the stress level as perceived by the students. Where nearly half of the students (50.0\% and $49.8 \%)$ had moderate academic and personal stress respectively, whereas, around two fifth (42.5\% and $37.3 \%)$ of the students suffering from moderate social and transportation related stress respectively. Finally around half $(48.3 \%)$ of the students have moderate total stress level.

Figure 3 portrays the coping strategies used by the students, religious related strategies comes firstly with a mean of $84.7 \pm 24.1$ followed by acceptance, self-blaming, positive reframing and planning strategy with a mean of $66.1 \pm 32.5,62.8 \pm 32.4,62.1 \pm 29.6$ and $61.3 \pm 29.5$ respectively. While venting, denial and behavioral disengagement reported by less than forty percent of the students (39.7 $\pm 28.6,38.6 \pm 32.7$ and $38.4 \pm 28.7$ respectively).

Figure 4 portrays that more than half $(52 \%)$ of the students had fair coping strategies, compared to one quarter(25\%) who classified as poor coping strategies.

Table 8 shows a significant between types and level of stress among the students, academic, inter- personal, social or transportation related stress. Where there is a significant differences observed between students, academic, personal, social or transportation and coping strategies among the undergraduates’ students. 
Table 9 shows a significant difference between age, gender and family income and the level of stress among the students, where there is a significant observed between gender and coping strategies among the students.

Table 10 shows a significant between working and academic experience of students with level of stress and coping strategies, where there was a significant differences observed between students working in night shifts and their level of stress. On the other hand there was a significant differences observed between academic level of students during last term and their coping strategies.

Table 11 spectacles the multivariate analysis logistic regression for stress level and coping strategies among undergraduates' students, it was observed that family income affecting the stress level by (26.075 times) followed by gender (11.301 times) and body mass index (BMI) (3.755 times) with a statistical significant relation. Additionally, gender affecting the coping strategies by (12.809 times) followed by body mass index (BMI) (4.829 times) and living with family (3.794 times) with a statistical significant relation.

\section{Discussion}

Stress in nursing education is the foremost crucial problems within the present time. It may be distinctive as a twentieth century disease and has been viewed as a posh and dynamic between individual and their environments. Furthermore it may be considered a psychological threat during which the individual perceives a situation as a potential threat [15].

Nursing students are susceptible to stress because of the nature of faculty life. High levels of stress are believed to have an effect on student health and educational functions. If the stress isn't dealt effectively, Feelings of loneliness, nervousness, sleeplessness and worrying might result. Knowledge of the stressors and their harshness among nursing students can be obliging in effective management and counseling of the students on how to cope and adapt to stressor. [16]. So the current study aimed to illustrate level of stress and identifying the coping strategies among students at Faculty of Nursing, Damanhour University.

Unwelcome stress happens in circumstances with a great level of external burdens in mixture with a low level of individual control. This is possibly the case in overloaded departments and therefore explains why the students experienced an increased level of stress there. The existing study give a clear picture of 400 nursing students their age ranged from 19-23 years. They were nominated from four academic years both boys and girls. On the topic of residence two thirds of the students were living in urban and majority of them living with their family and travel from residence to faculty, 51.5\% took more than 50 minutes followed by $29.5 \%$ travel 30 to 50 minutes and $19 \%$ spend only $<30$ minutes. These explain that highest mean percent of stressors in current study observed in stress related transportations. This is strike down with study done in china by Zhao, $\mathrm{F}$ et al., [17] who found that stress from assignment and workload was one of the major stresses experienced by more than 300 nursing students.
Parents have certain value system and norms which they want their sibling to track, students experienced academic stress arising from both their own expectations to excel as well as expectations arising from their parents. The incidence of reported parental pressure differed by parental education levels and their occupation .Academic stress may be due to pressure of parents for academic achievement $[18,19]$. The current study displayed that two thirds of students had obtain excellent and very good score in their academic performance although more than half of parents had diploma education it may be attributed to that parents from lower and middle class social strata want their children to do well in studies since this is often the only means to an honorable vocation for them .These findings are consistent with findings from a study done by Deb et al., [20] who reported that $60 \%$ of students experienced academic pressure mainly from their parents

Challenges are a part of nursing education and whether they are taken positively or negatively depend on countless factors like personal resources, social support available, study skills, transition to clinical curriculum, staying away from home, change of language, working environment, interpersonal conflicts, personal competence and work overload etc. All this leads to lifestyle changes like lessened leisure and recreational activity, reduced physical activity and sleep deprivation which then has an impact on the emotional well-being of them. High level of stress may have a negative effect on cognitive functioning and learning of students in nursing collages [21,22].

The present study revealed that minority of the students reported health problems like allergy in the nasal sinuses ,headache and abdominal pain on the other hand , more than half of the studied faculty students suffered from psychological stress, anxiety ,depression , sleeping disorders and low self-esteem. It may be attributed to nursing students susceptible to long hours of study, frequent examination and assignments, fear of failure, frequent contact with debilitated and dying patients. These finding come to an agreement with study done in Nepal [23] which specified that about half of nursing students suffer from psychiatric problems. Besides the results be at variance with another study done by Risal etal., [24] Which detailed that only few students had psychological problems as disturbed sleep and anxiety.

The current study itemized that about half of the students had moderate stress level related to academic, inter personnel and social stressors while nearly quarter of them had severe stress. It may be attributed to multiple reasons as nearly less than half of students in this study did not had availability of play and recreational activities and these findings showed that nursing students as they are newly exposed to a clinical environment mutual with the stress of completing new unfamiliar assignments and procedures could have higher stress. Other investigations identified clinical sources of stress, such as lack of knowledge and professional ability, lack of familiarity within history and medical terms, being in an unfamiliar situation, and mistakes with patients or handling of technical instruments [25,26].

This finding is consistent with the findings of previous studies accompanied in India by kumar R [27] out of 180 nursing students nearly one third of them had moderate 
stress whereas $32.8 \%$ had high stress respectively. In contrary to these findings a study done in India by Vajrala B et al., [28] who discovered that nursing students experience high levels of stress. Moreover study done by Daengthern L [29] who definite that 2nd and 3rd year students during their clinical practice were at high level of stress $53.2 \%$, followed by $22.7 \%$ at moderate level. Conversely, stress level was mild among Greek student nurses and this mild stress level is helpful to the individual's health, particularly when confronted with challenge and responsibility [30].

This study found that there is a significant differences between some demographic variables and overall perceived stress level among students as age, gender, family income and students worked in night shifts, some studies agreed with current results while other studies declared that this positive significant were found only between level of stress and age (Chan et al., 2009; Shaban et al., 2012) [31,32] In disparate to these finding study done in Saudi Arabia (2017) also found no significant differences in stress with regard to gender among undergraduate students [33].

In the present study, it was not a surprise that family income was a first significant indicator for students stress levels, as the income increased the level of stress reduced by 26 times. When tracing the profile of students, family income was enough in majority of them and helps for performing a paid activity such as research, semester work and even working "on duty shifts, while those in lower socioeconomic levels experience greater levels of stress and experience more stress-related health problems [34].

Likewise this current research found that the girls' gender has higher perceptions of stress compared to boys. Girls nursing students have an overall stress of $48.4+13.8$ which concurs with the result of the regression analysis. This means that the female gender among the other demographic variables is positively associated with the perceived stress of the nursing students with odd value of 11.301. This is harmonized by Bonneville-Roussy [35] where girls in universities experienced higher levels of stress and mostly affected by disengagement-oriented coping. In study conducted in Menoufiya University, Egypt [36] stress was significantly associated with gender, residence, perceived socioeconomic standard, feeling loneliness, and sharing family in social activities.

Additionally the current study discovered that age had a significant effect on students' stress level. The nursing students of age group less than 21 might perceive stress more than those of age group more than 21 due to younger students tend to experience emotional symptoms as a response to stress compared to that older students. This may be due to fact that as students get older, they expanded not only better knowledge and clinical expertise but also with problem solving skills and stress defensive strategies crucial when confronted with various stressors. This result is familiar with the study of Bam et al., [37] who found that the older nursing students enrolled in the nursing program for a period of time have a tendency to have a lower level of stress.

When the smoking behavior was associated positively with higher consumption of coffee, [38] the finding of present study disagree with that in which the majority of student did not smoke and nearly half of them drink coffee daily. It may be due the habitual consumption has been significantly related to increased self-reported alertness, improved performance of tasks and fewer intervals of attention, and improved long-term memory [39]. Shepard JD et al., [40] reported similar findings in a study on nursing students, and showed that both the quantity and frequency of caffeine use increased from the first year to the final year of the curriculum. It was amazing that coffee intake was a significant indicator for students stress level by 3.5 times in this study.

Some studies found positive association between obesity and psychiatric morbidity (depression and/or anxiety), especially among girls, but others didn't find association between obesity and mental health [41,42]. The present study, demonstrated that more than half of students were overweight and obese; it was not astonishment that body mass index (BMI) was a momentous indicator for students stress levels, a higher stress scores was detected among obese and overweight than normal students. These findings are in accordance with some findings of study performed in Jizan University which conveyed that factors such as BMI showed statistical significant differences with stress [43]

Shift work is considered critical to safeguard stability of care in hospitals. Expressly, the night shift is one of the most frequent causes for the disturbance of daily rhythms, causing major alterations of sleep and biological functions that can affect physical and psychological well-being and negatively impact on work productivity [41]. The current study given away that nearly fifth of students working in private hospitals all year, more than three quarters of them had night shifts and more than half of them had more than one shift per day. Statistical significant difference was observed among students who had night shifts and their stress level. These results in line with study done on Italy by Ferri P etal., [44] which propose that RNS night shift work signifies a stress factor which can lead to chronic discomfort and mild somatic alterations. It may be due to night shift work can frequently induce sleep disorder which can denote a risk factor for poor mental and physical health predisposing to the development of psychological and physical diseases.

Coping strategies imply the specific efforts both behavioral and psychological, that people employ to master, reduce tolerate or minimize stressful events. Coping with stress for a student nurse is a dynamic and ongoing process, aimed at survival, growth and maintenance of the individual integrity. Coping is a momentous mediator of stress and illness. Nursing students should develop active strategies for coping with the new stressors that they experience. Studying coping strategies in nursing students and their contributing factors is therefore necessary for performing any kind of primary intervention directed to them [45].

Present study publicized that more than half of students use fair coping strategies while one quarter of them using poor coping. It may be attributed to that nearly two thirds of students in this study reported academic and job satisfaction. Other studies on nursing students have also confirmed the relationship between academic satisfaction and positive coping strategies [46]. Another study done by Khater et al., [47] also confirmed the lower levels of stress among nursing students who were interested in studying 
nursing and participants who were more sociable and had better communication skills stated that this attribute made them better cope with clinical stress.

Additionally, the students in the current study were found to use different coping strategies to cope with stress. Majority of the students $84.7 \%$ used religious coping mechanisms and $66.1 \%$ of them used acceptance strategies for coping mechanism. The nursing students were found to use self-blame, positive reframing, planning, active coping mostly rather than behavioral disengagement, self-distraction and denial .It might be because it is easy to be cope using emotional strategies than problem based strategies for their comfort. This finding is reliable with a recent study done in Negeria [48] and study done by Sami-Abdo et a (10.4\%) in Malaysia [49]. On the other hands, Study from Japan [50] recounted that medical students often resort to alcohol, tobacco and drug use as a coping strategy, these discrepancy as the result of culture and religious factors between two countries. In opposition, Shaban et al., [32] found that avoidance was the most commonly used mechanism among students in dealing with different stresses. Avoidance is generally familiar as only a temporary measure and not a maintainable solution to address underlying stressor. Furthermore, in a Taiwanese study done by Demir et al., [51] staying optimistic was more frequently used than other coping strategies. Other coping strategies stated in study done by Rinsky et al., [52] that include effective communication strategies, prevention of management conflicts and perceived control.

Additionally, the study done in India [53] displayed that most commonly used strategy by the students was ventilating feelings as complaining to their friends (36.7\%) and crying $(22.8 \%)$, listen to music, stereo or radio (36.7\%),engaged in day-dreaming (23.3\%) hobby (14.1\%) eat food $(13.9 \%)$ try to think of or see good things (26.7\%), try to organize their life (19.4\%) and try to make their own decisions (18.3\%).Around 32\% of the students tried to cope with the stress by helping other people in solving their problems or by blaming others for their problems respectively.

The current study reported that there was no significant difference between coping strategies and age, place of residence, living with family and family income. However, the gender and academic performance were significant association feasible to student to cope with stress. Moreover, gender, BMI and living with family were good predictors for coping strategies Chan et al., [31] found that the main predictor of coping mechanism was years of study and religion.

Awareness of the stressors and their severity among nursing students in the nursing training faculty can be helpful in effective management and counseling of the students on how to cope and adapt to stressors. This results information is also useful for nursing management and nursing teachers to identifying students' needs, effective planning, facilitating their learning in the academic and clinical setting, to reduce or prevent stress during their education and training. Additionally, strengthening nursing students' positive coping skills may be helpful for them to effectively deal with various stressors during their educational experiences while maximizing learning [54].

\section{Conclusion}

It has been concluded that half of the nursing students had felt moderate stress due to transportations, academic, interpersonal and social factors. It was found that the perceived stress level was statistically significant with age, gender, family income and students worked at night shifts. Further, the nursing students were found to use religious and acceptance for coping rather than denial and behavioral disengagement.

\section{Recommendations}

- The findings of this study indicate a need for stress management programs in all nursing faculties as an immediate measure to address stress, and its effectiveness with consideration of families and peers as well as nursing students themselves

- Conduct workshops on stress and effective coping strategies through the academic years. The presence of counsellors among the faculty may help students overcome stressful conditions.

- Nursing educators and curriculum designers should consider the optimization of curriculums to offer a less stressful experience for students (e.g. ensuring low-intensity academic requirements when students undertake clinical placements

- Establish a counselling center in each university to deal with psychological problems confronted students.

- Further research should explore the particular factors exacerbating the experience of stress among students relative to curriculum design.

\section{Acknowledgements}

We would like to thanks all the students who participated in the study for their valuable time given for filling the questionnaires.

\section{Competing Interests Statement}

The authors declare that there are no competing or potential conflicts of interest.

\section{References}

[1] Ninaus K, Diehl S, Terlutter R, Chan K, and Huang A. Benefits and stressors - Perceived effects of ICT use on employee health and work stress: An exploratory study from Austria and Hong Kong. Int J Qual Stud Health Well-being. 2015; 10.

[2] Samina Y, Hussain M, Parveen K, Gilani S. Coping Strategies of Nursing Student against Academic and Clinical Stress at Public Sector Lahore. Int. J. Soc. Sc. Manage. 2018; 5 (3): 209-218.

[3] Waled A.M, Ahmed B, Mohammed M.A. Nursing students' stress and coping strategies during clinical training in KSA. Science direct. 2019.

[4] Mustafa M, Illzam EM,. Muniandy RK,.Hashmi MI, Nang MK. Causes and Prevention of Occupational Stress .IOSR Journal of Dental and Medical Sciences. 2015; 14 (11) Ver. VIII: 98-104.

[5] Karihe J, Namusonge I. Effects of Working Facilities Stress Factors on the Performance of Employees in Public Universities in 
Kenya. International Journal of Scientific and Research Publications. 2015; 5(5). ISSN 2250-3153.

[6] MingLiu K, Thomas K.S, Wong M, Luo Z, Chan M. Perceived stress among Macao nursing students in the clinical learning environment .International Journal of Nursing Sciences. 2015; 2 (2); 128-133.

[7] Sossah L, Asiedu Y. Stress management and resilience in junior and senior nursing students in Ghana. European Journal of Research and Reflection in Educational Sciences. 2015; 3 (2) 2.

[8] Dragana D, Golubović B, Brkić N, Prokeš B. Professional stress and health among critical care nurses in Serbia. 2012; 63: 171-180.

[9] Jameson, P. R. The effects of a hardiness educational intervention on hardiness and perceived stress of junior baccalaureate nursing students. Nurse education today, 2013; 34(4), 603-607.

[10] Khater, W., Akhu-Zaheya, L., \& Shaban, I. Sources of stress and coping behaviors in clinical practice among baccalaureate nursing students. International Journal of Humanities and Social Science.2014; 4(6).

[11] Shah M, Hasan S, Malik S, Sreeramareddy CT. Perceived stress, sources and severity of stress among medical undergraduates in a Pakistani medical school. BMC Med Educ. 2010; 10:2.

[12] Chadalawada UR, Matli P. Assessment of stress among medical college students of Government Siddhartha Medical College, Vijayawada, Andhra Pradesh, India. Int J Med Sci Public Health. 2016; 5(6): 1240-124.

[13] Uehara T, Takeuchi K, Kubota F, Oshima K, Ishikawa O. Annual transition of major depressive episode in university students using a structured self- rating The Egyptian Journal of Community Medicine.2010; 35(2).

[14] World Health Organization. Global Database on Body Mass Index. 2011 [Online] available at; www.apps.who.int/bmi/ index.jsp.

[15] Carver CS. You want to measure coping but your protocol's too long: Consider the brief COPE. Int J Behav Med. 1997; 4(1): 92100 .

[16] Alzayyat A. Study about the stress among nursing students during clinical education. 2014

[17] Zhao, F.-F., Lei, X.-L., He, W., Gu, Y.-H., \& Li, D.-W. The study of perceived stress, coping strategy and self-efficacy of Chinese undergraduate nursing students in clinical practice. International Journal of Nursing Practice. 2015; 21(4), 401-409.

[18] Zeetha M , Shantibala K, Akoijam B , Vizovonuo M, Vanlalduhsaki M. Perception of Students on Parental And Teachers' Pressure on Their Academic Performance. IOSR Journal of Dental and Medical Sciences.2018; (11) 17: Issue 01 Ver. I .68-75.

[19] Mukherjee P. J. Kids driven to breaking point: Pushed by parents to be all-rounders, the perform-or-perish pressure on school kids is turning them into nervous wrecks. 2010.

[20] Deb S, Strod E, Sun J. Academic Stress, Parental Pressure, Anxiety and Mental Health among Indian High School Students. International Journal of Psychology and Behavioral Sciences.2015; 5(1), 2015: 26-34.

[21] An H, Chung S, Park J, Kim S-Y, Kim KM, Kim K-S. Noveltyseeking and avoidant coping strategies are associated with academic stress in Korean medical students. Psychiatry Res. 2012; 200:464-468.

[22] Sandover S, Jonas-Dwyer D, Marr T. Graduate entry and undergraduate medical students' study approaches, stress levels and ways of coping: a five year longitudinal study. BMC Medical Education. 2015; 15: 5.

[23] Lama S , Shrestha M .Pandey A, Shyangwa P, Deo B. Psychological problems among nursing students: study from a Nepalese health institute. European Psychiatry .2013; 28(1).

[24] Risal A, Sanjel S, Sharma PP. Study of Depression among the Nursing Students in a University Medical College of Nepal. Kathmandu University Medical Journal. 2017 14(55): 264-8.

[25] Pulido-Martos M, Augusto-Landa J, and Lopez-Zafra E, “Sources of stress in nursing students: a systematic review of quantitative studies,” International Nursing Review. 2012; 59 (1): 15-25.

[26] Labrague L. Stress, stressors, and stress responses of student nurses in a government nursing school. Health Science Journal .2014; 7(4).424-435.

[27] kumar N. Stress and Coping Strategies among Nursing Students. Nursing and Midwifery Research Journal. 2011; 142-146.
[28] Vajrala B, Debora J. Level of Stress and Coping Strategies among B. Sc. Nursing2018 Students. The International Journal of Indian Psychology.2018; 6 (3).

[29] Daengthern L. Factor influencing stress among nursing students of faculty of nursing during clinical practice. 2014.

[30] Papazisis G, Tsiga E, Papanikolaou N, Vlasiadis I, and SapountziKrepia D. Psychological distress, anxiety and depression among nursing students in Greece. International Journal of Caring Sciences. 2008; 1 (42).

[31] Chan C, So W\& Fong D. Hong Kong Baccalaureate Nursing Students' Stress and Their Coping Strategies in Clinical Practice. Journal of Professional Nursing.2009; 25(5): 307-313.

[32] Shaban I. A., Khater, W. A., \& Akhu-Zaheya, L. M. Undergraduate nursing students, stress sources and coping behaviors during their initial period of clinical training: A Jordanian perspective. Nurse Education in Practice. 2012; 12(4), 204-209.

[33] Alsaqri S. Stressors and Coping Strategies of the Saudi Nursing Students in the Clinical Training: A Cross-Sectional Study. Education Research International . 2017; Article ID 4018470, 8.

[34] Sarokhani D, Delpisheh A, Veisani Y, Sarokhani MT, Manesh K, Sayehmiri K. Prevalence of depression among university students: a systematic review and meta-analysis study. Hindawi Publishing Corporation Depression Research and Treatment. 2013.

[35] Bonneville-Roussy A, Evans P, Verner-Filion J, Vallerand R, Bouffard T. Motivation on and coping with the stress of assessment: Gender differences in outcomes for university students. Contemporary Educatonal Psychology. 2017; 48: 28-42.

[36] Abdallah AR, Gabr HM. Depression, anxiety and stress among first year medical students in an Egyptian public university. Int Res J Med Med Sci .2014; 2(1): 11-9.

[37] Bam V, Oppong G, and Ibitoye M. Stress and coping mechanisms of nursing students during clinical practice in Ghana. Journal of Science and Technology. 2014. 34(2): 50-59.

[38] Freedman N. D., Park Y., Abnet C. C., Hollenbeck A. R., Sinha R. Association of coffee drinking with total and cause-specific mortality. N Engl J Med. 2012; 366: 1891-904.

[39] Larson C \& Joubert G. Medical students' use of caffeine for 'academic purposes' and their knowledge of its benefits, sideeffects and withdrawal symptoms. 2014 ; 51(4):322-327.

[40] Shepard JD, Al'Absi M, Whitsett TL, et al. Additive presser effects of caffeine and stress in male medical students at risk for hypertension. Am J Hypertens. 2010; 13:475-81.

[41] Ibrahim MB, Abdelreheem MH. Prevalence of anxiety and depression among medical and pharmaceutical students in Alexandria/University Faculty of Medicine, Alexandria University. Alexandria J Med .2015; 51: 167-73.

[42] Zhao G, Ford ES, Dhingra S, Li C, Strine TW, Mokdad AH. Depression and anxiety among US adults: associations with body mass index. Int J Obes 2009; 33: 257-266.

[43] Sani M, Mahfouz MS, Bani I, Alsomily AH, Alagi D, Alsomily NY. Prevalence of stress among medical students in Jizan University, Kingdom of Saudi Arabia. Gulf Med J. 2012; 1 (1): 19-25.

[44] Ferri P, Guadi M, Marcheselli L Balduzzi S, Magnani D, and Lorenzo R. The impact of shift work on the psychological and physical health of nurses in a general hospital: a comparison between rotating night shifts and day shifts. Risk Manag Healthc Policy. 2016; 9: 203-211. Published online 2016 Sep 14.

[45] D’Souza MS, Venkatesaperumal R, Radhakrishnan J, Balachandran S. Engagement in clinical learning environment among nursing students: role of nurse educators. Open J Nurs. 2013; 3: 25-32.

[46] Hirsch CD, Barlem ELD, Almeida LKd, Tomaschewski-Barlem JG, Figueira AB, et al. Coping strategies of nursing students for dealing with university stress. Revista Brasileira deEnfermagem 2015. 68: 783-790.

[47] Khater W, Akhu-Zaheya L, Shaban I . Sources of stress and coping behaviors in clinical practice among baccalaureate nursing students. Int J Human Soc Sci. 2014; 46: 194-202.

[48] Oku A, Owoaje E, Oku O, Ikpeme B. Prevalence of stress, stressors and coping strategies among medical students in a Nigerian medical school. African Journal of Medical and Health Sciences. 2018; 12. 
[49] Abdo S, Redhwan A, Mustafa A, Krishna G. Stress and Coping Strategies of Students in a Medical Faculty in Malaysia. Malaysian J Med Sci. 2011; 18: 57-64.

[50] Watson R, Watanabe K, Yamashita A, Yamaguchi M. A Japanese version of the stressors in nursing students (SINS) scale science direct.2018; 5(2), 181-185.

[51] Demir E, Bektaş T and Laporte G. A review of recent research on green road freight transportation. European Journal of Operational Research. 2014; 237(3): 775-793.

[52] Rinsky JL, Nadimpalli M, Wing S, Hall D, Baron D, Price LB,
Larsen J, Stegger M, Stewart J, Heaney CD . Livestock-associated methicillin and multidrug resistant Staphylococcus aureus is present among industrial, not antibiotic-free livestock operation workers in North Carolina. Plos one. 2013; 8(7): e67641.

[53] Bista B1, Bhattrai B2 and Khadka N Stress and Coping Mechanisms among nursing students in Kathmandu.2017; 3(1) ISSN: 2091-1041 I.

[54] Labrague L, McEnroe D,Alexis J, Santos D, Olaide B. Examining stress perceptions and coping strategies among Saudi nursing students: A systematic review Nurse. 2018; 65 192-200.

(C) The Author(s) 2019. This article is an open access article distributed under the terms and conditions of the Creative Commons Attribution (CC BY) license (http://creativecommons.org/licenses/by/4.0/). 